L e o, Zeitschrift f. physiolog. Chem. Bd. 469, 490.

Lebedeff, Pflügers Arch. für d. gesamte Physiologie Bd. XXXI. 1883.

Lusenna, Sperimentale IV. 1903.

Perls, Lehrbuch der allgemeinen Pathologie. 1877.

Pettenk ofer und Voit, Zeitschrift f. Biologie, IX.

Ribbert, Centralbl. f. allgem. Pathologie. 1893.

Rosenfeld, Berliner klinische Wochenschr. 1904 (22-23).

Stolnikow, Du Bois-Reymonds Archiv für Anatomie und Physiologie. Suppl.-Bd. 1887.

Vo it, Physiologie des allgemeinen Stoffwechsels. Hermann, Handbuch der Physiologie, VI. 1881.

Ziegler und Obolensky, Zieglers Beiträge, II. 1888.

\title{
XVIII.
}

\section{Ein Enterokystom des Mesenteriums und Netzes.}

(Aus dem Pathologisehen Institut der Universität Greifswald.)

Beitrag zur Kenntnis der cystischen Abdominaltumoren.

Von

Gertrud Roegner,

cand. med.

(Hierzu Taf. XIV.)

Die Lehre von den cystischen Mesenterialgeschwülsten gehört in der allgemeinen und speziellen Geschwulstlehre zu den am wenigsten erforschten Gebieten. Die Ursache liegt wohl hauptsächlich an dem überaus seltenen Vorkommen dieser Tumoren, dann aber auch in der sehr schwierigen Deutung der histologisehen Befunde. Ebenso schwierig kann sich die Entscheidung der Frage gestalten, ob es sich bei der Entwicklung dünnwandiger Cysten im Mesenterium um primäre oder secundäre Vorgänge handelt, besonders wenn derartige Cysten dureh Operation mit folgender Heilung gewonnen wurden, ein jeden Zweifel beseitigender Sektionsbericht also fehlt. Beschäftigen wir uns zunächst mit der Frage der primären Geschwülste, so kommt als Matrix in erster Reihe in Betracht das System der Lymphgefäße. Georg Wegner hat 
Virchoms Archin, Bd CldXXX

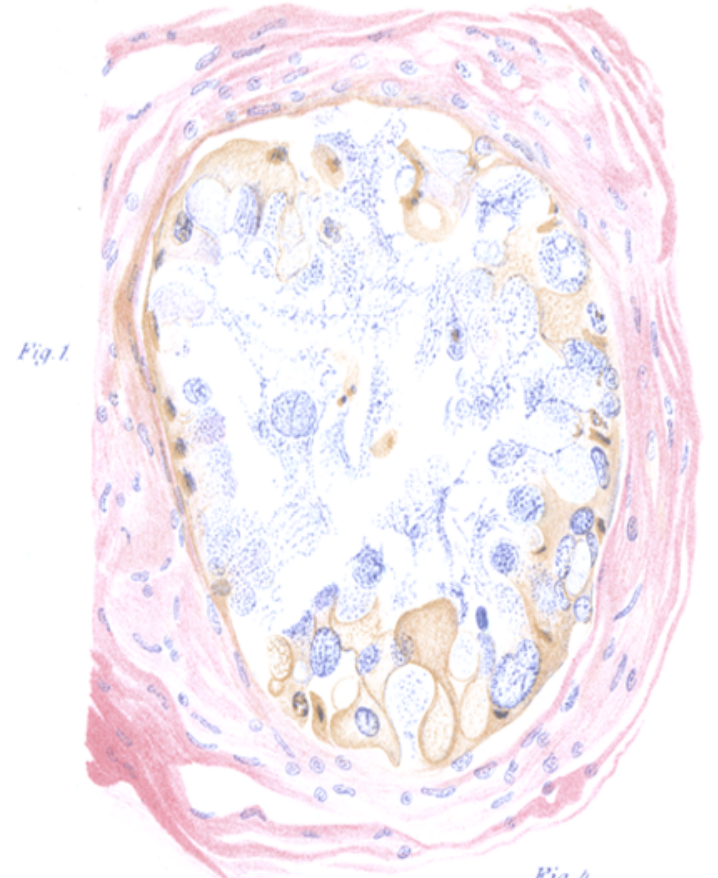

Pig. A.
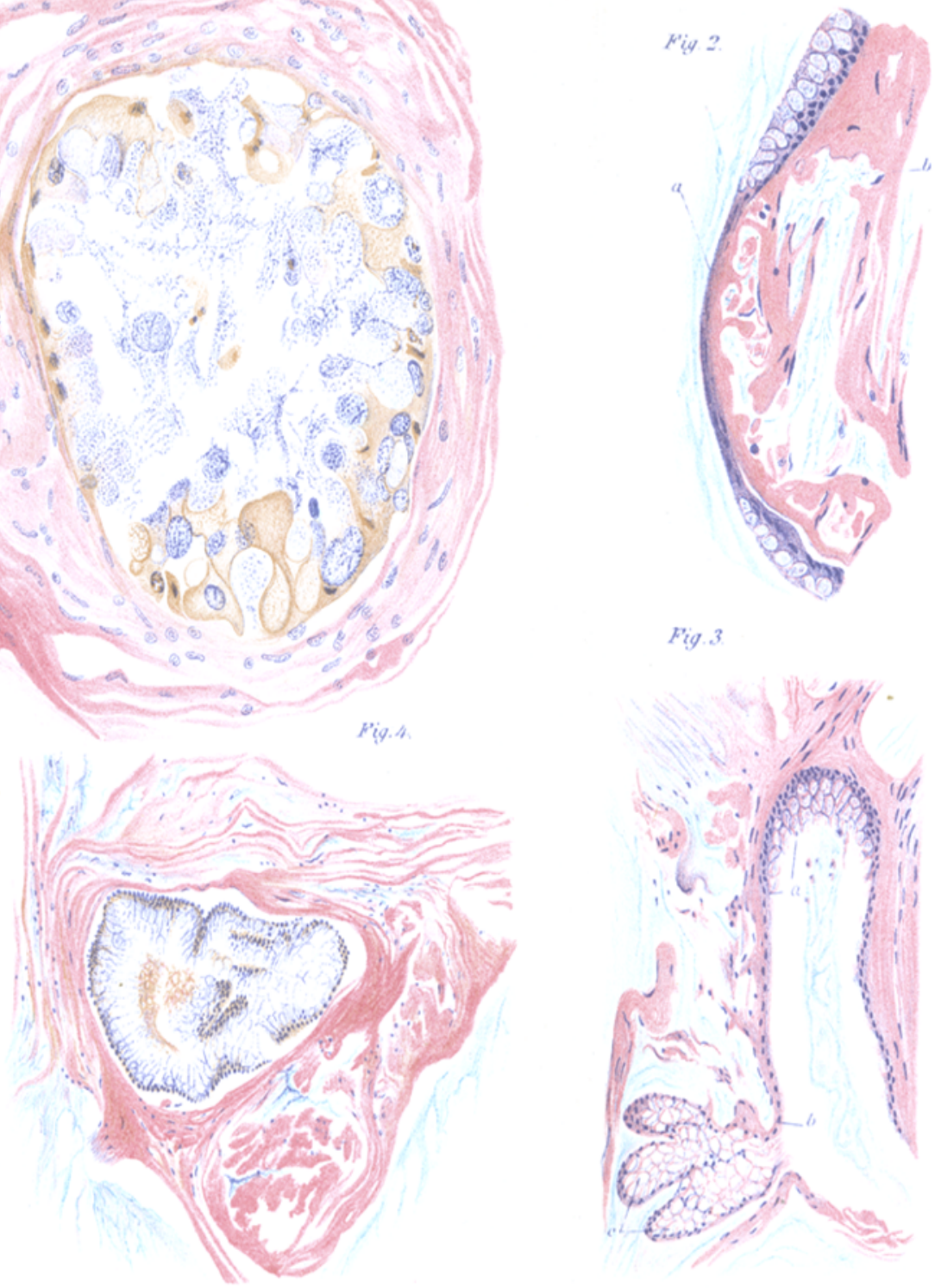

Fig. 3

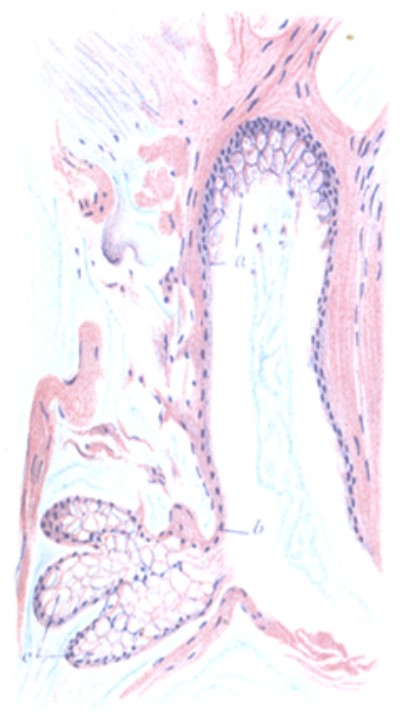


seinerzeit beschrieben, wie durch allmähliche Erweiterung und Vermehrung von Lymphgefäßen diejenige Form der Neubildung entstehen kann, die er als "Lymphangioma cysticum" bezeichnet hat. Von ihm an Tieren durch fortgesetzten starken Luftdruck künstlich erzeugt, finden sich in der Literatur bis in die neveste Zeit doch auch immer wieder Fälle beschrieben, bei denen auf natürlichem Wege Cysten in abdomine entstanden waren, von mikroskopisch kleinen bis zu beträchtlich großen Hohlräumen, deren Inhalt Lymphe, deren Wandauskleidung Endothel ist. Dagegen ist die Frage, ob und inwieweit das Bindegewebe sich am Prozesse der Lymphangiomwucherung beteiligt, eine zurzeit noch viel umstrittene.

Typische Lymphangiome wurden in neuester Zeit von Penkert in so ausführlicher und klarer Form beschrieben, daß es für das Verständnis meiner weiteren Ausführungen vielleicht vorteilhaft ist, hier zunächst die von Penkert beschriebenen Eigenschaften eines Lymphangioms (Fall 1) in knappester Form noch einmal als typisch hervorzuheben: Der Inhalt der Cysten und Lymphräume ist durchweg dünnflüssig, teils wasserklar, teils, da es sich um Kombination des Lymphangioms mit Hämangiom handelt, mit Blut gemischt, teils rein blutig. Das Aussehen des Inhalts unter dem Mikroskope ist durchweg homogen, an einzelnen Stellen von der Wand abgehoben, erscheint er als homogene Vacuole. Vielfach, zum Teil massenhaft, schwimmen in dem homogenen Inhalt weiße und rote Blutkörperchen. Die Wandauskleidung besteht entweder aus typischem, durchaus unverändertem Endothel, oder sie fehlt ganz und die Cyste ist unmittelbar von Binde- oder Fettgewebe umschlossen, nirgends aber, in keinem der von ihm beobachteten und sonst noch erwähnten Fälle ist eine Endothelwucherung im Sinne epithelialer Umwandlung zu beobachten. Das umgebende Binde- und Fettgewebe zeigt einen großen Kernreichtum, d. h. es ist in Mitwucherung begriffen, außerdem enthält es in den vorgerückteren Teilen der Geschwulst glatte Muskulatur.

Nun kommen aber ganz seltene Cystengeschwülste im Mesenterium und Netz vor, die sich von diesem Typus der Lymphangiome weniger durch ihr äußeres Aussehen, als durch den Cysten- 
inhalt und vor allem durch die mikroskopischen Bilder unterscheiden und daher als Gruppe für sich betrachtet werden müssen.

Ein Fall dieser Art ist kürzlich dem Greifswalder Pathologischen Institut zur Untersuchung. eingesandt worden. Der Bauchtumor wurde am 31. März 1905 im Johanniterkrankenhause in Polzin von dem Leiter der Anstalt, Herrn Sanitätsrat Dr. Schmidt, exstirpiert. Das Begleitschreiben des Herrn Dr. Stübinger lautete wörtlich:

„Das anbei übersandte Präparat stammt von einer 69 jährigen Frau. Dieselbe hatte angeblich seit einem Jahre eine Geschwulst im Leibe bemerkt, welche erst in letzter Zeit schneller gewachsen sein sollte. Das angeblich langsame Wachstum, die geringen Allgemeinerscheinungen, der sonstige Befund sprach für ein Ovarialkystom. Die Laparotomie ergab eine Cyste, die dem Mesenterium aufsaß, Colloid enthielt. Die Cystenwand war nach oben vom entarteten, geschwulstigen Netz bedeckt. Ein Stück Netz ist excidiert worden und zur histologischen Bestimmung vielleieht von Wichtigkeit."

Die von Herrn Geheimrat Prof. Dr. Grawitz sofort vorgenommene Untersuchung des frischen Präparates ergab den folgenden Befund, den nachträglich für meine Arbeit niederzuschreiben Herr Prof. Grawitz die Güte hatte:

„,,Von den größeren Blasen, durch deren dünne fibröse Wand, ähnlich wie beim multiloculären Ovarialkystom, glasiger Inhalt durchschimmert, wird eine eröffnet. Der Inhalt ist so zäh, daß nur ein Teil herausquillt, der nach seiner bläulich weißen Farbe, seinem meist transparenten Aussehen and nach seiner Konsistenz mit Stärkekleister verglichen werden kann. Vielfach ziehen sich opak weiße Streifen durch die homogenen Massen hindurch. Mikroskopisch ist die Substanz großenteils homogen, enthält aber wellige Fäden, die aus feinsten albuminösen Körnchen zusammengesetzt sind, hier und da Haufen von Zell- oder Kernbröckeln, ohne charakteristische Formen. Von der Innenfläche der Wand werden an mehreren Stellen zusammenhängende äußerst zarte Häutchen abgeschabt; diese zeigen sehr schöne, regelmäßig polygonale, saftreiche platte Zellen, die im frischen Zustande an das Epithel der Froschhornhaut erinnern. An einzelnen Schnitten durch das von kleinsten Cystchen durchsetzte Fettgewebe des Netzes läßt sich eine epitheliale Umkleidung der homogenen Schleim- oder Gallertklümpchen nicht nachweisen, diese scheinen vielmehr die Gewebsmaschen in Form sehr verschieden groBer Einlagerungen zu durchsetzen.

Eine definitive Diagnose konnte aus diesen Bildern nicht gestellt werden. Das einzige, was zweifellos erschien, war 
das, daß der zähe Inhalt als das Sekretionsprodukt schleimbildender Zellen resp. als Produkt einer schleimigen Degeneration anzusehen war. Kurz vorher war hier ein Fall von cystischer Ausweitung im blinden Ende eines proximal'verschlossenen Wurmfortsatzes zur Beobachtung gekommen; der blasig erweiterte Abschnitt enthielt' zähen Schleim, der mit dem hier vorliegenden 'vollkommene Übereinstimmung zeigte, und da im Netz so viele kleine Schleimklümpchen, von äuBerst zarten Häuten überzogen, vorlagen, wurde an die Möglichkeit gedacht, daß etwa wie in dem von Eugen Fränkel mitgeteilten Falle der Inhalt eines zerplatzten Wurmfortsatzes in die Bauchhöhle entleert and am Netze angeklebt und umwuchert sein könnte.

Um zur Klarheit zu kommen, wurde bei Herrn Sanitätsrat Schmidt in Polzin angefragt und beim Einlegen der Präparate in Formalin die Aufgabe gestellt, innerhalb der größeren und kleineren Cystchen nach dem secernierenden Epithel zu suchen. " i"

Auf unsere Anfrage in Polzin ging uns alsbald in dankenswertester Weise über Anamnese, Krankheitsverlauf und Operation der folgende Bericht zu:

„Witwe D., 69 Jahre alt, hat $10 \mathrm{mal}$ geboren, 8 Kinder leben und sind gesund. Alle Geburten und Wochenbetten normal verlaufen. Außer Gelenkrheumatismus, der ohne Residuen und ohne Herzaffektion ablief, hat Pat. keinerlei Krankheiten durchgemacht.

Vor etwa 2 Jahren merkte sie, daßs der Leib stärker wurde. Dabei stellten sich auch zeitweise ziemlich heftige Schmerzen, besonders in der Kreuzgegend ein. Mit dem allmählichen Wachstum der Geschwulst traten auch häufiger Harndrang und Obstipation auf. Im übrigen war das. Allgemeinbefiaden nicht gestört, vor allem keine Kachexie vorhanden. Ende März, kam sie zur Operation ins Krankenhaus, die am 31. März 1905 unter Chloroformnarkose vorgenommen wurde (Herr Sanitätsrat Dr. Schmidt).

Abdominalgeschwulst: colloidales Cystom vom Mesenterium des Dünndarms ausgehend mit etwa $6 \mathrm{~cm}$ breitem Stiel. Netz mit miliaren opalescierenden Knötchen besetzt, ebenso der Mesenterialstumpf. Ein Teil des Netzes wird zur histologischen Untersuchung excidiert. Die mehrkammerigen Cysten lassen sich nicht in toto entfernen, sondern platzen bei der Entwicklung, einige sind schon vorher geborsten, da Colloid frei in der Bauchhöhle schwimmt. Eine Verwachsung des Tumors mit dem Netz ist nicht vorhanden; die kleinen Knötchen (Cystchen), die Netz, Mesenterium und Stiel der 'großen Geschwulst bedecken, erstrecken 
sich nicht auf den Dünndarm, der ein völlig normales Aussehen bietet. Von dem Mesenterium wird der Stiel der Cystengeschwulst nach Unterbindung der einzelnen Gefäße durch Scherenschlag: getrennt, der Stumpf wird vernäht und versenkt. Die Bauchhöhle wird durch Peritonaealnaht, Faltennaht und besondere Hautnaht geschlossen, vor völligem Schluß läßt man $500 \mathrm{ccm}$ physol. Kochsalzlösung von $40^{\circ} \mathrm{C}$. Temperatur einlaufen. Airolpuderung der Nähte, Heftpflaster zum Abschluß, steriler Verband.

Die Heilung verlief ungestört bisher. Die Laparotomiewunde hat sich gut geschlossen. Der Zustand der Frau ist beŕriedigend."

Soweit der ärztliche Bericht vom 17. April 1905. Der in Formalin gehärtete groBe, dick wurstförmige, mehrkammerige Cystentumor hat eine Länge von $20 \mathrm{~cm}$, Querdurchmesser $7 \mathrm{~cm}$, Umfang $17,5 \mathrm{~cm}$ und ist prall gefüllt mit einer dick gallertigen, teils glasig durchscheinenden, teils getrübten Masse. An dieser großen Cyste hängen zahlreiche kleine, runde, von Walnuß- bis StecknadelkopfgröBe, alle mit dem gleichen Inhalt erfüllt. Ebenso ist das mitgesandte Stückchen Netz übersät von kleinen Cysteben und in toto verdickt.

Die mikroskopische Untersuchung wurde an zehn verschiedenen Blöcken von den verschiedensten, räumlich weit getrennten Cysten, teils vom Mesenterium, teils vom Netz stammend, vorgenommen. Die Härtung erfolgte in Formalin, Einbettung in Paraffin, Färbung mit Hämateïn-Eosin und - zum weitaus größten Teile - nach van Gieson mit HämateïnVorfärbung. Mehrere mikrochemische Proben auf spezifische Schleimfärbung (Sublimatbehandlung, Thionin) ergaben negative Resultate. In den säntlichen anderen Präparaten zeigt der Inhalt der zum Teil mikroskopisch kleinen Cysten eine blauviolette Hämateïnfärbung, ist aber nirgends homogen, sondern durchweg zu welligen Linien, oft bis zur Kreisform, oder zu netzartiger Struktur angeordnet. Der Zusammenhang: mit der Cystenwand ist überall ein sehr loser, nur an wenigen Stellen auf längere Strecke ununterbrochen, meistens nur ans mehr oder weniger starken Schleimfäden bestehend, häufig völlig unterbrochen.

Innerhalb dieser Masse finden sich zahlreiche Zellelemente, vereinzelte Spindelzellen, meistens große polygonale Zellen, zum Teil mit noch wohlerhaltenem Kern und Kernkörperchen. An anderen Stellen erscheint der Kern degeneriert, die Zelle selbst als eine matt tingierte Protoplasmamasse. Noch wieder an anderen Stellen liegen ganze Zellkomplexe im. Cysteninhalt, teils reihenförmig angeordnet, teils in ungeordneter Masse, und gerade diese letzteren zeigen die sonderbarsten Zellveränderungen: Das ganze Gebilde erscheint gequollen, blasig, der Kern sowohl wie das Protoplasma, das eine überans deutliche Schaumstruktur zeigt; stellenweise fehlt in diesen großen Zellen der Kern auch ganz und das Protoplasma scheint in völliger Auflösung begriffen zu sein.

Die einzelnen Cystchen und Cysten sind ursprünglich offenbar ringsum geschlossen, wenigstens kann man schon unter der Lupe beobachten, wie sie, in den verschiedensten Ebenen getroffen, doch einen 
ringsum durch Bindegewebe geschlossenen Hohlraum bilden. Bei dem allmählichen. Wachsen aber werden die einzelnen Septa vielfach immer dünner und reißen schließlich ein, so daß eine Kommunikation und schließliches Zusammenfließen mehrerer Cystchen stattfindet. Wir haben uns also die größeren Cysten entstanden zu denken einmal durch die fortgesetzte Sekretion der ursprünglichen, dann aber aus der Verschmelzung mehrerer Cystchen.

Die Cystenwandung ist teils rein bindegewebiger Natur, teils zeigt sie einen äußerst polymorphen Zellbelag. Von ganz platten, endothelähnlichen Zellen sind alle Formen vertreten bis zum hohen Zylinderepithel mit radiär gestellten Kernen. Was aber noch viel überraschender ist: ich fand an zahlreichen Stelien mitten im hohen Zylinderepithel typische Becherzellen, ja ganze Reihen von Zylinderzellen erscheinen in Becherzellen umgewandelt und in starker Sekretion begriffen. (Fig. 1 u. 2 Taf. XIV.) Auch die nicht typischen Becherzellen, Formen, die den Eindruck etwas gequollener Zylinderzellen machen, gehen vielfach ohne scharfe Zellgrenze in den Cysteninhalt über (Fig. 4, Taf. XIV), waren also ebenfalls in Sekretion begriffen. Sehr schön ausgeprägt sind an vielen Stellen die von den secernierenden Zellen ausgehenden Strömungslinien. die dort, wo es zur Ab- und Auflösung ganzer Zellverbände kommt, vielfach eine netzartige Struktur annehmen, so daß man die Zellkonturen noch sehen kann, ohne daß von Protoplasma und Kern noch etwas vorhanden wäre. Lösen sich nun zum Teil schon ganze Reihen von Zylinderzellen ab, um als zusammenhängende Masse in das Lumen der Cyste zu fallen (Fig. 4, Taf. XIV), so ist das noch weit anschaulicher der Fall bei den Becherzellen. Wir finden hier eine solche Hypersekretion, eine so wilde Wucherung der einander drängenden und oft in mehrfacher Lage übereinander befindlichen, teils geschlossenen, teils weit offenen Becherzellen, daB es den Auschein hat, als gingen sie, selbständigen Wesen gleich, die jede Herrschaft über sich verloren haben, in wilder Unordnung der völligen Auflösung entgegen. (Fig. 1, Taf. XIV.) Und zwar ist diese enorme Zellwucherung am stärksten ausgeprägt in den ganz kleinen Cystchen, während der Epithelbelag der größeren ein viel ruhigeres Aussehen bietet oder, wie sehr häufig; in Form so platter dünner Zellenhärtchen den Cystenraum auskleidet, daß es vielfach den Anschein hat; als ob er in den Profilbildern der großen Cystenquerschnitte gänzlich fehlte. Inwieweit bei den mehr kubisch und flach erscheinenden Epithelzellen Schiefschnitte in Betracht kommen, ist oft schwer zu entscheiden. Jedenfalls finden sich auch merkwürdig flache Becherformen, so daf wir hier Schiefschnitte mit ziemlicher Sicherheit annehmen können. Höchst interessant ist die an manchen Präparaten (Fig. 2 u. 3, Taf. XIV) zu machende Beobachtung, wie die Epithelien ihre Zellform entsprechend den Konturen der Cystenwand verändern: Da, wo diese in einem nach dem Lumen zu konkaven Bogen verläuft, finden wir die schönsten und höchsten Zylinder- und Becherformen; wird die Linie gestreckter, so 
nehmen die Zellen einen melr kubischen Typus an, um sich ganz abzuflachen, wo es über konvexe Vorsprünge geht. Der Zellbelag wird hier oft ganz endothelartig oder verschwindet scheinbar an vielen Stellen ganz. Dieser Befund stimmt mit dem oben erwähnten überein, daß der hohe Epithelbelag am schönsten in den kleinen Cystchen zu sehen ist, während er anscheinend in den großen oft ganz fehlt: In beiden Fällen findet eine Abplattung und allmähliches Schwinden des Epithelbelags statt an den Stellen zunehmender und gröBter Spannung, ein Befund, der für die Deutung des Ganzen von prinzipieller Bedeutung ist. (Fig. 2 ․ 3, Taf. XIV.)

In dem die Cysten umschließenden Gewebe fallen drei Dinge sofort in die Augen: Das stellenweise reichlich eingelagerte Fettgewebe, die pralle Füllung aller Blutgefäße, auch der kleinsten Kapillaren, und die eigentümlich zerklüftete, wie oedematöse Beschaffenheit des ganzen Bindegewebes. In den Wandungen der Cysten fanden wir bei genauerer Untersuchung mehrfach glatte Muskelfasern, am schönsten ausgeprägt in der Wand des großen Tumors (Block V), wo sämtliche glatte Muskelfasern im Querschnitt getroffen sind mit deutilichem, hier natürlich rund erscheinendem Kerne.

Zwischen die Bindegewebszüge eingelagert fanden sich zahlreiche einzelne Nester großer, polygonaler Zellen, oft nur zu 2 bis 3 aneinander gelagert, mehrfach aber auch hier schon deutliche Becherform und Secretion zeigend, an anderen Stellen zu ganzen Zellreihen angeordnet nach Art von Drüsenschläuchen oder in Bildern, wie sie im Querschnitt getroffene Drüsengänge aufweisen. Dazwischen fanden sich wieder ganze Spalten oder runde Cystchen völlig auskleidet mit regelmäßigem Zylinderepithel, allemal aber mehr oder weniger mit Spuren von Secretion und dem gleichen Inhalt wie in den typischen Cysten. Daß ein Teil dieser zahlreichen, im Bindegewebe klaffenden Spalten mit oedematösem Gepräge Lymphspalten sind, ist wohl sicher, denn sie finden sich ja allenthalben auch im normalen Gewebe, nicht aber können wir sie durchweg als solche anerkennen, besonders nicht diejenigen kleineren und oft schon beträchtlich großen Spalten, deren Wand einen typischen Epithelbelag mit allen vorher geschilderten Eigenschaften hat, einen Epithelbelag, der in allen tieferen schmalen Buchten so typisch wird, daß man echte Drüsen zu sehen glaubt (Fig. 3); auch können wir nicht als Lymphspalten diejenigen Stellen anerkennen, wo ganz vereinzelte secernierende Zellen sich findèn, deren jede sofort einen kleinen Hohlraum, mit secernierter Flüssigkeit erfüllt, schafft und somit wohl wesentlich zu dem oedematösen Aussehen des Ganzen beiträgt. Sehr charakteristisch in dieser Beziehung ist wiederum die Wand der großen Cyste, die bereits wegen ihres Muskulaturgehaltes erwähnt wurde: Hier findet sich innerhalb des fibrösen Gewebes einmal eine nur mikroskopisch sichtbare Cyste, deren Zellen aber von allen, die wir sahen, die stärkste Verwirrung aufweisen (Fig. 1), und dicht daneben findet sich ein längerer Spaltraum, in dessen Inhalt sich massenhafte Bänder und 
Züge von großen Zellen finden, die offenbar von einer ehemals secernierenden Wandbekleidung stammen, nun aber, losgelöst, in ihrem eigenen Produkt dem Untergange entgegengehen. Vielfach finden sich auch kleine Einstülpungen des Bindegewebes in das Lumen der Cyste, oft nur als ganz dünne Septa, und da wo, diese mit regelmäßigem. Epithel bekleidet sind, erhalten wir das Bild typischer Papillen. In manchen Präparaten enthält die Wand eine vollkommen papilläre Struktur, so daß eine vollendete Ähnlichkeit mit Ovarialcystomen vorliegt. Solche von Zylinderepithel überzogenen zarten Papillen, wie Fig. 4 nur eine einzige zeigt, liegen in andern Cysten in groBer Zahl nebeneinander.

Im übrigen trifft man im Gewebe stellenweise eine mäßige, kleinzellige Infiltration, die nur, soweit wir bisher finden konnten, an zwei Stellen zu einer herdweisen Anhäufung geführt hat, ähnlich den Noduli lymphatici, aber ohne deutlich wahrnehmbares Keimzentrum.

\section{Epikrise und Diagnose.}

Wenden wir uns nun der Frage nach Art und Entstehung. dieses cystischen Tumors zu: der bei dem ersten Anblick auftauchende Gedanke an ein multiples Lymphangiom trat, je weiter unsere Untersuchung vorrückte, immer mehr zurück. Eine kurze Gegenüberstellung der eingangs erwähnten prägnantesten Eigenschaften eines typischen Lymphangioms (I) einerseits und unseres Tumors (II) andererseits wird es als berechtigt erscheinen lassen, die Gruppe der Lymphangiome für unsern Fall differentialdiagnostisch auszuschließen.

I.

1. Inhalt überall dünnflüssig.

2. Inhalt homogen in den gehärteten und gefärbten mikroskopischen Präparaten.

3. In der homogenen Masse viele Leukocyten, keine Zellmassen oder Zelldetritus.

4. Die Wandauskleidung besteht ausschließlich aus Endothelzellen oder fehlt ganz.
II.

1. Inhalt dick, gallertig, zäh.

2. Inhalt nirgends homogen, sondern wellen- und netzförmig geordnet.

3. Leukocyten sind nirgends zu finden, um so mehr epitheliale Zellen und Zellverbände, z. T. in deutlicher Auflösung begriffen.

4. Als Wandauskleidung sind alle Zellformen von großen Becher- und Zylinderzellen bis zu ganz flachen, endothelartigen, zu finden. 
5. Eine Secretion von seiten der Wandungszellen ist nirgends zu beobachten, Zellen und Inhalt sind getrennt.

6. Das umgebende Binde- und Fettgewebe ist in Mitwucherung begriffen und zeigt an vielen Stellen eine zellige, die Bindegewebssepta begleitende Infiltration.
5. Fast sämtliche Zellen sind in starker Secretion begriffen und gehen mit ihrem Inhalt zum großen Teil ohne scharfe Zellgrenze in den Inhalt der Cyste über. Das Hervorgehen des schleimigen Inhalts aus den Becherzellen ist in allen Stadien fixiert und so sicher zu beobachten wie in normaler Darmschleimhaut.

6. Eine Wucherung des umgebenden Gewebes ist nicht zu konstatieren. Eine ganz geringe, kleinzellige Infiltration findet sich nur an sehr vereinzelten Stellen.

Schwieriger gestaltet sich schon die Entscheidung der Frage, ob es sich hier vielleicht um Karzinom handelt. Die Anamnese ergab, daß die Patientin seit zwei Jahren Beschwerden hat. Die erste Entstehung des Tumors ist wahrscheinlich noch um einen weiteren Zeitraum zurück zu verlegen, denn die Beschwerden der Frau, Harndrang, Obstipation und Kreuzschmerzen, lassen sich alle auf mechanischen Druck von seiten des immer größer werdenden Tumors zurückzuführen, während das Allgemeinbefinden ein ungestörtes war. Ferner sind bei der Laparotomie an anderen Bauchorganen keine krankhaften Veränderungen, keine Metastasen wahrgenommen worden, trotzdem sogar schon Cysten geplatzt waren und ihr Inhalt frei in der Bauchhöhle lag.

Die Geschwulstbildung war durchweg cystischer Natur; nirgends eine destruktive Neigung zeigend und absolut auf Mesenterium und Netz beschränkt. Bei einem solchen Berunde würden wir eine maligne Neubildung mit Sicherheit ausschließen müssen, wenn es nicht bekannt wäre, daß es Gallertkrebse giebt, die ein außerordentlich protrahiertes Wachstum mit nur 
geringen Allgemeinerscheinungen haben. In der Tat zeigen auch die mikroskopischen Bilder stellenweise eine überraschende Ähnlichkeit mit Gallertkrebs, besonders an den Stellen, wo das Bindegewebe durch die secernierte Flüssigkeit und die erweiterten Lymphspalten zerklüftet erscheint und einzelne große polygonale Zellen und Zellnester birgt. Außer diesen höchst verdächtig aussehenden Stellen finden sich aber zahlreiche andere, deren Bau so vollständig von dem aller bisher untersuchten Gallertkrebse verschieden ist, daß wir, zugleich in anbetracht des klinischen und makroskopischen Befundes Karzinom im strengen Sinne ausschließen zu dürfen glauben. Daß der Tumor nicht absolut gutartig ist, zeigt sein stetig, wenn auch sehr langsam fortschreitendes Wachstum; eine zerstörende Neigung im Sinne typischer Karzinome fehlt, dafür ist die Secretion eine um so größere, und sie ist offenbar laut Krankheitsbericht im letzten Jahre immer reichlicher geworden, so daß es sich fragt, ob nicht eine ausgesprochene Bösartigkeit die alsbaldige Folge gewesen wäre, hätte die Trägerin des Tumors sich nicht einer Operation unterzogen.

Müssen wir also Lymphangiom und Gallertkarzinom ausschließen, so weist uns die Anwesenheit der secernierenden Epithelien, vor allem der Becherzellen, auf den Darm hin und damit auf eine dritte Gruppe cystischer Abdominaltumoren, der Enterocystome. Die erste eingehende Beschreibung von Fällen dieser Art wurde durch Roth gegeben und veröffentlicht. Roth wies bei seinen Cysten teils noch einen Zusammenhang mit dem Darmlumen nach, teils war dieser schon aufgehoben, es fanden sich aber in der Cystenwand alle Schichten der Darmwand wieder. Der Inhalt ergab Mucinreaktion. Teilweise aber war der Charakter der Darmwand nicht mehr gewahrt, ja es fanden sich ganz glattwandige Cysten, deren Entstehung Roth selbst auf eine frühere Entwicklungsstufe zurückführt. In allen Cystenwandungen war Muskulatur nachweisbar, Roth läßt aber die Frage offen, ob es auch muskellose Wandungen giebt.

$\mathrm{Da}$ es zu weit führen würde, alle bisher beschriebenen Fälle von Enterocystom zu erwähnen, obwohl ihre Zahl eine durchaus beschränkte ist, so sei hier nur noch die Arbeit von 
Runkel erwähnt, welcher 20 Fälle dieser Art, einschließlich der Rothschen zusammengestellt hat. In allen Fällen zeigt die Cystenwand mehr oder weniger deutliche Darmstruktur, eine von Nasse beschriebene wird ausdrücklich als muskellos bezeichnet in den rom Darme am meisten entfernten Teilen, der Inhalt der Cysten wird fast durchweg als dickflüssig, z. T. als zäher Schleim beschrieben. Ausnahmslos handelt es sich um jugendliche Personen, meistens Kinder, in zwei Fällen (Rimbach) waren die Trägerinnen der Tumoren 24 und 38 Jahre alt, was als besondere Merkwürdigkeit angesprochen wird. In eben diesen beiden Fällen war aber auch die Darmstruktur der Cystenwand am stärksten verändert, die Wand „bestand aus Bindegewebe, in das regellose Züge von Muskelfasern eingestreut lagen, die innere Auskleidung erinnerte noch an atrophierte Darmschleimhaut," "stellenweise war die Darmschleimhaut durch derbfaseriges Bindegewebe ersetzt". In beiden Fällen war die Cyste auch nicht mehr in so unmittelbarer Verbindung mit dem Darm, sondern saß an einem mehrere Zentimeter langen Stiel dem Dünndarm auf, eine Kommunikation von Darm und Cyste konnte nicht nachgewiesen werden. Diese beiden Fälle geben entschieden zu denken, die Schlußfolgerung möchte ich mir aber noch vorbehalten bis nach der Besprechung einer dritten einschlägigen Arbeit.

Es ist die Abhandlung von Nager „Beitrag zur Kenntnis seltener Abdominaltumoren", und da Citate daraus hier viel zu weit führen würden, kann ich nur auf die ganze Arbeit und die für die Beurteilung meines Falles wichtige Ähnlichkeit des Züricher Falles mit dem meinigen verweisen. Ich muß gestehen, daß diese Arbeit von Anfang bis Ende eine einzige große Ủberraschung für mich war. Denn es handelt sich bei dem darin beschriebenen Fall des Züricher Pathologischen Instituts offenbar um ein vollkommenes Analogon zu dem unsrigen. Anamnese und Krankheitsverlauf, makroskopisches wie mikroskopisches Aussehen entsprechen einander in hohem Maße, mit dem einzigen Unterschied, daß wir neben den Zylinderzellen auch Becherzellen fanden und an einzelnen Stellen glatte Muskulatur nachweisen konnten. Die Übereinstimmung 
erstreckt sich aber nicht auf die Deutung der Tumoren, da Nager für den seinigen die Diagnose auf "Lymphangioendotheliom" stellt and in ausführlicher Weise diese Auffassung zu. begründen sucht.

Bei der großen Aufmerksamkeit, welche die junge Lehre von den Endotheliomen, insbesondere derjenigen der Lymphwege, allenthalben erregt, and in anbetracht der zahlreichen Irrtümer, die auf diesem Gebiete bereits nachgewiesen werden konnten, sei es mir gestattet, etwas näher auf die Nagersche Auffassung unserer gleichartigen Tumoren einzugehen.

Eine physiologisch-chemische Untersuchung des frischen Cysteninhalts ist bei uns leider unterblieben. Angeregt durch die Arbeit Nagers; machte ich nun aber den Versuch, auch bei dem bereits mit Formalin behandelten Präparate zu einem Resultat bezüglich der chemischen Beschaffenheit des Cysteninhalts zu kommen. Der Direktor des hiesigen physiologischen Instituts, Herr Professor Dr. Bleibtreu, hatte die große Liebenswürdigkeit, die Untersuchung auszuführen: Das Resultat war abermals genau dasselbe wie im Falle Nagers: Im Cysteninhalt konnte Mucin nicht nachgewiesen werden, wohl aber Eiweißkörper, wie sie ähnlich auch in der Lymphe vorkommen. Auch die mikrochemische Prüfung auf Mucin ergab ein negatives Resultat. Trotzdem sind wir weit entfernt, mit Nager zu sagen: Folglich ist der Inhalt eingedickte Ly mphe!

Es müßte dann zunächst einwandfrei nachgewiesen werden, daß es sich hier um genau dieselben Eiweißverbindungen handelt wie in der Lymphe. Das konnte aber bisher nicht geschehen, es können nur Körper nachgewiesen werden, die sich ,wie Pseudoglobulin“ verhalten und die Eigenschaften "eines Albumins“ zeigen, während andere wichtige Bestandteile der Lymphe, z. B. Fibrinogen, Euglobulin, nicht nachgewiesen werden konnten.

Wir können in unseren mikroskopischen Präparaten, wie ausführlich beschrieben wurde, Becherzellen in großer Zahl nachweisen. Da es im ganzen menschlichen Körper nur schleimsecernierende Becherzellen giebt, müssen wir annehmen, daß Schleim, wenn auch vielleicht nur in sehr kleinen Mengen, doch im Cysteninhalt vorhanden oder vorhanden 
gewesen ist. Mucin ist die Verbindung eines Eiweißkörpers mit einem Kohlehydrat, es erscheint uns bei den unter so sonderbaren Verhältnissen lebenden Zellen durchaus möglich, ja wahrscheinlich, daß auch ihr Produkt von vornherein vom normalen Mucin etwas abweicht und sich um so eher durch einen sich unserer Kenntnis entziehenden chemischen Vorgang eine Abspaltung des Kohlehydrats gefallen lassen muß. Ein solcher Vorgang erscheint um so eher möglich, wenn wir die physiologisch-chemische Beschaffenheit des Cysteninhalts noch weiterhin - nicht untersuchen - sondern ruhig überlegend betrachten. Ausführlich ist beschrieben worden, wie nicht nur einzelne Zellen, sondern ganze Zellverbände sich loslösen, in das Cysteninnere fallen und dort einfach vergehen. Nun sind aber „Eiweißkörper die einzigen Stoffe, die ausnahmslos mit Sicherheit in jeder Zelle gefunden werden" (Verworn), durch die massenhafte Auflösung von Zellen muß also der Cysteninhalt an Eiweißgehalt gewinnen. Ferner aber scheinen auch alle Zellen, nicht nur die Becherzellen, in Secretion begriffen zu sein, wenigstens spricht Dr. Nager es gleich mir direkt aus, „dab sich das Zellprotoplasma vom Cysteninhalt nicht scharf trennen läßs". Was aber geht hier aus den Zellen in das Lumen der Cyste über? Protoplasma? Das würde abermals gleichbedeutend sein mit Eiweiß. Oder andere Körper, vielleicht Fermente, die das von den Becherzellen secernierte Mucin zerlegen?

Wir wissen ferner von anderen, besser erforschten pathologischen Erscheinungen im Körper, z. B. den Ovarialeystomen, daß ihr ursprünglich fast reiner Mucininhalt außerordentlich eiweißreich werden kann durch Transsudation aus dem umgebenden Gewebe infolge mechanischer oder entzündlicher Reizung; ebenso verhält es sich mit dem reichen Eiweißgehalt entzündeter Schleimbeutel.

Daß sich in den Cysten aber z. T. vielleicht auch echte Lymphe findet, halten wir durchaus nicht für unmöglich, ohne deshalb anzunehmen, daß die Geschwulst darum von den Endothelien der Lymphbahnen ausgegangen sein müsse. Ich erinnere hier an das, was bei der mikroskopischen Beschreibung über das oedematöse Aussehen des Bindegewebes und seinen 
Grund gesagt wurde. Nehmen wir an, daß irgendwo im Bindegewebe des Mesenteriums fremdartige, bisher ruhende Zellen aus irgendeinem Anlaß ihre Tätigkeit, hier also Secretion, zu entwickeln anfangen, so müssen sie für ihr Secret erst Platz schaffen, denn die Natur hat ihnen keinen reserviert, d. $h$. mit andern Worten: ihr Secret wird in alle Lücken, in alle Spalten im Bindegewebe eindringen, diese überall erweitern, dehnen, Bindegewebsfasern auseinanderdrängen and, wo es auf bereits vorhandene Flässigkeit triftt, sich mit dieser auf das innigste vermischen. Lymphspalten finden sich aber überall im Gewebe, so wird auch im Cysteninhalt ein Teil vielleicht echte Lymphe sein.

Wir möchten hier, bei der Erklärung des oedematösen Aussehens des Bindegewebes, sogar noch einen Schritt weiter gehen und es teilweise auch mit dem Endothel in Verbindung bringen, da, wo von deutlichem Endothel überhaupt die Rede sein kann. Die allgemeine Physiologie hat uns gelehrt, daß es keine lebende Zelle gibt, die nicht auf Reizung hin mit einer Steigerung der ihr eigentümlichen Funktion reagierte. Wir wissen ferner aus Heidenhains Untersuchungen, daß auch die Endothelzellen einer actir secernierenden Tätigkeit fähig sind. Nach unserer Auffassung ist durch die Anwesenheit fremder, tätiger Zellen mit so sehr starker Secretion, wie es unsere mikroskopischen Präparate zeigen, ein Reiz für die in der Nähe gelegenen Endothelien gegeben, stark genug, um auch sie zu einer gesteigerten Tätigkeit anzuregen. Damit ist aber wiederum eine Vermehrung von Lymphe, eine Vermehrung des Eiweißgehaltes der alle Gewebslücken füllenden Flüssigkeit gegeben. Wir können daher nicht mit Nager die Neubildung als eine Folge einer Lymphspaltenaffektion ansprechen, sondern betrachten vielmehr die von epithelialen Entodermzellen ausgehende Bildung als das Primäre und die, übrigens nicht sicher bewiesene Affektion der Endothelien als das Secundäre. Nirgends aber hat uns die Physiologie bisher berichtet, daß gereizte Zellen sich auch in morphologischer Beziehung so zu verändern vermöchten, wie Nager es hier und andere Beobachter es an andern Körperstellen den Endothelzellen zutrauen, daß nämlich Lymphgefäßendothelien durch Steigerung ihrer Funktion etwa 
in typische, schleimabsondernde Zylinder- und - in unserem Falle - in Becherzellen verwandelt werden könnten.

Hiermit gelangen wir aus dem physiologisch-chemischen Gebiet auf das der Histologie and Entwicklungsgeschichte.

Bei der Frage, ob es sich um eine LymphgefäBendothelwucherung handelt oder nicht, ist ein Punkt ganz besonders auffallend: die ausschließliche Beschränkung der Neubildungen auf Mesenterium und Netz. Die Lymphbahnen des Mesenteriums stehen aber in engster und unmittelbarer Verbindung mit den umgebenden Organen, namentlich dem Darm. Wir wissen aus der histologischen Anatomie, daß jede Darmzotte ihr axiales Chylųsgefäß hat, daß dieses in direkter Verbindung steht mit den Saftlücken und Lymphspalten des umgebenden Bindegewebes und durch diese wieder mit den größeren Lymphbahnen. Ginge nun die Geschwulstbildung vom Endothel dieser Lymphstraßen aus, so wäre die Freilassung der Darmserosa usw. in der Tat eine äußerst merkwürdige Erscheinung, wie sie ja auch Nager ganz besonders aufgefallen ist. Ganz hinfällig erscheint uns dabei die Vermutung; "daß die Lymphbahnen in den Grenzgebieten von Mesenterium und Darmserosa vielleicht andere Verhältnisse böten", es müßten dann wenigstens auch Vermutungen darüber ausgesprochen werden, inwiefern die Verhältnisse der Lymphbahnen hier plötzlich andere sein sollten, oder die Ansicht, „daß die Geschwulst zeitlich noch nicht so weit vorgeschritten sein dürfte"! Geschwülste, die zwei bis zehn Jahre lang wuchern und dabei am Orte ihres Ursprungs eine so weitgehende Entwicklung aufweisen, wie es in unsern beiden Fällen zu beobachten ist, sollten in diesem langen Zeitraum noch nicht Zeit gefunden haben, auf den für sie von der Natur geschaffenen Straßen, die auch ihren Ursprung bilden, bis zur Darmserosa vorzudringen? Das erscheint uns ganz ausgeschlossen, und gerade dieses merkwürdige Haltmachen der Wucherung an den Grenzen ihres Entstehungsgebietes läßt eine primäre Neubildung von Endothelien der Lymphwege als mindestens sehr zweifelhaft erscheinen.

Nun aber zur Frage der Umwandlung der einzelnen Endothelzelle selbst. Eine ganze Reihe von Forschern traut ihr die Fähigkeit zu, aus einer Bindegewebszelle zu einer Zylinderzelle 
zu werden, und man will Bilder dieser Art zweifellos gesehen haben. Wir haben nun in unserm Tumor zum ersten Male, soweit wir die Literatur der Mesenterialkystome kennen, neben den Zylinderzellen auch Becherzellen nachgewiesen. Gesteht man die Möglichkeit der Umwandlung von Bindegewebszelle in Zylinderzelle zu, so wird man vor der Becherzelle nicht gut Halt machen können, sondern sagen müssen: eine Bindegewebszelle kann auch schleimsecernierende Becherzelle werden. Auf grund aber aller bisherigen histologischen und embryologischen Forschung and bei der Dunkelheit, die noch immer das Gebiet der Cellularphysiologie umgibt, werden wir vielleicht gut tun, uns an Linnés Wort zu erinnern: Natura non facit șaltus. Man erlaube mir eine kleine Exkursion in das Gebiet der Entwicklungsgeschichte.

Aus dem primären inneren Keimblatt entwickelt sich 1. das Darmdrüsenblatt oder secundäre innere Keimblatt, 2. die Chordaanlage, 3. die beiden mittleren Keimblätter, das Mesoderm. Von diesen letzteren, die auch als parietales und viscerales Mittelblatt oder als Hautfaserblatt und Darmfaserblatt bezeichnet werden, gehen alle Mesenchymkeime aus; welche die Grundlage für die ganze Gruppe der Bindesubstanzen bilden und, aus ihrem ursprünglichen epithelialen Verbande losgelöst, alsbald ein in sich selbständiges Fül]gewebe darstellen, dessen Zellen nirgends mehr, weder morphologisch noch funktionell, an die epitheliale Abkunft erinnern.

So klar die Embryologie diese Verhältnisse gelegt hat, so sehr läßt sie uns noch im Dunkeln bei der Frage der Entstehung von Gefäßendothelien. Hier stehen drei Theorien nebeneinander. Die erste (Ziegler) läßt Blut- und Lymphgeïäße aus Resten der primären Leibeshöhle hervorgehen, also aus Zwischenräumen zwischen den beiden primären Keimblättern, die bei der wachsenden Ausbreitung des Mesenchyms von diesem als Lacunen, Interstitien, Gefäße umschlossen werden.

Die zweite Auffassung sucht den Ursprung der Gefäße in anfänglich von Mesenchymzellen gebildeten soliden Zellschläuchen, die allmählich einen Hohlraum zwischen sich schaffen, in der Weise, daß die inneren Zellagen zu Blutkörperchen, die äußeren dagegen zur Endothelwand werden. 
Diesen beiden Theorien, die übereinstimmend eine rein mesenchymale, d. h. rein bindegewebige Natur der Endothelien annehmen, steht die dritte gegenüber, die, einen getrennten Ursprung von Bindesubstanzen und Gefäßendothel nebst Blut vertretend, das gesamte Stützgewebe aus dem Mesenchym, Endothelien aber aus den Zellen des Darmdrüsenblattes hervorgehen läßt durch Abschnürung eines Endothelsäckchens aus dem Epithel des inneren Keimblattes und Bildung des Gefäßbaumes durch Sprossung.

Eine ausführlichere Darlegung des verwickelten Kapitels der Endothelentstehung würde den Rahmen dieser Arbeit überschreiten. Die gedrängte Ubersicht, in unmittelbarer Anlehnung an Hertwigs Entwicklungsgeschichte gegeben, mag genügen, um im weiteren zwei wichtige Punkte nicht aus den Augen zu verlieren: Entweder ging die Zellwucherung unseres und auch des Züricher Tumors tatsächlich von Zellen der Gefäßwandung aus. Dann aber würden wir hierin eine kräftige Unterstützung der dritten Theorie sehen: Endothelien sind gar keine Bindegewebszellen, sondern epithelialen Ursprungs vom secundären inneren Keimblatt her. Eine durch irgendwelche embryonale Störung veranlaßte ungenügende Umwandlung der aufgelösten epithelialen Zellverbände würde es dann leicht erklären, daß diese Zellen bei irgendeiner uns unbekannten Ursache plötzlich zu wuchern anfangen und dabei völlig ihren ursprünglichen Habitus zeigen. Es sind dann aber eben Zellen, die zwar zu Endothelien bestimmt waren, aber diese Umwandlung nie vollkommen durehgemacht, sondern den Charakter einfach versprengter Darmepithelien sich gewahrt haben.

Oder aber die Endothelien gehören in die echte Bindegewebsgruppe, wie es wohl die Mehrzahl der Forscher der Gegenwart annimmt. Dann aber möchten wir uns auf das Entschiedenste gegen eine Auffassung verwahren, die es für möglich hält, daß von allen Bindegewebszellen im Körper allein die Endothelien die bewunderungswürdige Fähigkeit besitzen sollen, hohes Zylinderepithel und - in unserem Falle - sogar große Becherzellen aus sich machen zu können. Es sei denn, man führte auch die Bindegewebszellen, auf ihren ersten Ursprung - die mittleren Keimblätter zurück, die 
sowohl kubische als zylindrische Zellen aufweisen, und das Mesoderm wieder auf seinen Ursprung, das Entoderm: so ist der Zirkel aber wiederum gesehlossen, denn dann handelt es sich eo ipso um versprengte Epithelien des inneren Keimblatts. Wir haben aber vorhin das ganze von Mesenchymkeimen abstammende Gewebe als ein in sich. Geschlossenes, absolut Selbständiges und von seinem Ursprung Unabhängiges bezeichnet und möchten daran auch festhalten.

Während also die progressive Metamorphose von Bindegewebszelle zur hohen Zylinder- und Becherzelle mindestens als sehr unwahrscheinlich und jedenfalls als noch ganz unerwiesen angesehen werden muB, ist die regressive Metamorphose von Zylinderepithel zu ganz flachen Zellen vielfach zu beobachten. Herr Geheimrat Prof. Dr. Grawitz hatte die Güte, mir anläßlich dieser Abhandlung einige besonders instruktive Präparate dieser Art zu zeigen. Es handelte sich um ein malignes Ovarialcystom, also ein Präparat, bei dem niemand bezweifeln wird, daß das hohe Zylinderepithel das.Primäre ist. Nun war in geradezu klassiseher Weise zu verfolgen, wie dieses Zylinderepithel in den tiefen Buchten der Cysten hoch und unberührt stand, im Verlaufe grader Linien flacher, kubisch wurde, da aber, wo es über Vorsprünge ging, sich sogar abplattete und so vollständig einen typischen Endothelbelag vortäuschte, daß es auch dem geübtesten Mikroskopiker ohne Kenntnis der Herkunft des Präparates unmöglich sein dürfte, diese vermeintlichen Endothelien als ursprüngliches, nur durch Dehnung abgeplattetes und degeneriertes Zylinderepithel zu diagnostizieren. Ich erinnere nun an die Beschreibung der mikroskopischen Bilder unseres Tumors; der Schluß liegt nahe genug, daß wir hier genau denselben Vorgang haben: Vollständige Abplattung, zum Teil völliges Zugrundegehen ursprünglicher Zylinder- und Becherzellen durch Spannung und Druck. (Fig. 2 u. 3, Taf. XIV.)

Es bleiben also als Matrix für unsern Fall mit größter Wahrscheinlichkeit nur embryonal versprengte Entodermkeime übrig. Es mag hier noch in aller Kürze Fall 4 und 5 der Runkelschen Arbeit zitiert werden. In beiden Fällen handelt es sich um kleinste Residuen des Dotterganges innerhalb 
der Wand des Ileum. Schleimhaut des Darmes normal, unter der Muscularis mucosae plötzlich mehrere, Drüsengängen ähnliche, von Zylinderepithel ausgekleidete Räume, nach diesen wieder eine völlig normale Muscularis und Serosa. Ganz gleiche Präparate weist auch unser Institut auf und es fragt sich sehr, ob derartige kleine kongenitale Unordnungen nicht vielleicht viel häufiger sind, als man bisher annahm, da sie ganz erscheinungslos bleiben können und somit häufig gar nicht oder wie in Fall 4 als zufälliger Nebenbefund entdeckt werden.

Schließen wir nun unsern Tumor den Enterocystomen an, d. h., nehmen wir nur einen graduellen Unterschied an, so ergibt sich die folgende Gliederung:

Die erste Gruppe umfaßt diejenigen Enterocystome, die auf einer verhältnismäßig späten Entwicklungsstufe entstanden sind, daher volle Darmstruktur zeigen, vielfach Kommunikation mit dem Darme, stark entwickelt sind, und entweder schon im Foetus, oder doch bald im frühen Kindesalter einen derartigen Charakter annehmen, daß sie den Tod herbeiführen, falls eine rasche Operation keine Rettung bringen kann.

Die zweite Gruppe, von Roth selbst schon auf ein früheres Entwicklungsstadium zurückgeführt, läßt die Bestandteile der Darmwand in ihren Cystenwandungen zwar noch erkennen, aber nicht mehr vollständig. Die Tumoren sind etwas weiter vom Darme entfernt, haben keine Kommunikation mehr mit ihm und entwickeln sich häufig erst ganz allmählich, daher ihre Träger ein höheres Alter erreichen können. Hierher gehören die oben besonders erwähnten beiden Rimbachschen Fälle.

Als dritte Gruppe reihen sich dann Fall 4 und 5 von Runkel an und diejenigen Fälle, von denen Präparate auch unser Institut besitzt. Fall 4 war, wie gesagt, ein zufälliger Nebenbefund bei einem Kinde, hatte also an sich noch gar keine Erscheinungen gemacht, bei Fall 5 handelt es sich um einen Erwachsenen ohne nähere Angaben.

Unser Fall nun würde eine vierte Gruppe eröffnen. Die Absprengung muß hier auf einem ganz frühen Stadium der Entwicklung erfolgt sein, jedenfalls wohl noch zur Zeit der flächenhaften Ausbreitung des: Embryo. Dafür spricht auch 
die vollständige Trennung der Cysten vom Darm und seinen Arihängen, die ausschließliche Lokalisation in Mesenterium und Netz. Die einzelnen Keime haben in ihrer fremden Umgebung zwei Menschenalter hindurch ruhen können, denn die Trägerin ist 69 Jahre alt. Die Frage ist von Bedeutung, ob nicht der Nagersche Tumor, dessen Träger ein Alter von 67 Jahren erreichte, ein noch etwas früheres Stadium repräsentiert, da weder glatte Muskelfasern noeh Becherzellen gefunden wurden, sondern einzig die kubischen und zylindrischen Zellen, wie sie nur Mesoderm und Entoderm aufweisen.

Die ganze Abhandlung wurde unter der Voraussetzung geschrieben, daß es sich um eine primäre Neubildung handelt, Ich möchte nicht unterlassen, zum Schlusse darauf hinzuweisen, daß die Frau, von welcher die Geschwulst entnommen wurde, noch lebt. Jedermann kennt die Überraschungen, welche eine der Operation folgende Sektion zu bieten vermag. Die Sektion fehlt hier aber, und damit der unanfechtbare Beweis, daß es sich um einen Primärtumor handelt, so außerordentlich unwahrscheinlich auch die Annahme eines anderen primären Herdes bis jetzt erscheint.

Bevor ich meine Arbeit aus den Händen gebe, drängt es mich, meinem hochverehrten Lehrer, Herrn Geheimrat Prof. Dr. Grawitz, meinen ehrerbietigsten, herzlichen Dank auszusprechen für die Ũbertragung dieser Arbeit und alle mir dazu gegebene gütige Anleitung. Ebenso danke ich an dieser Stelle noch besonders Herrn Professor Dr. Bleibtreu für die liebenswürdige Untersuchung des Cysteninhalts.

Erklärung der Abbildungen auf Taf. XIV.

Fig. 1. Mikroskopisch kleine Cyste aus der Wand des großen, dem Mesenterium anfsitzenden Tumors. Die sämtlichen Becherzellen sind in vollständiger Degeneration begriffen. Im linken oberen Teile beginnt der Zellbelag sich in toto abzulösen. Vergrößerung: Zeiß, Olimmers. Oeular 2. Technik: Hämatein-van Gieson.

Fig. 2. Wandpartie einer Cyste des Netzes. a) Vorsprung in das Innere mit vollkommen abgeplattetem Epithel, b) Spalträrme im stark 
zerklüfteten Bindegewebe. VergröBerung etwa 120 fach, Technik s. 0.

Fig. 3. Größerer Spaltraum im oedematösen Netz. a) intaktes Epithel, b) durch Spannung abgeplattetes Epithel, c) drüsenähnliche Bildung. Vergrößerung etwa 120 fach, Technik: Hämatein-Eosin.

Fig. 4. Kleine Cyste mit Zylinder- und Becherzellenbelag, der sich in toto von der Wand löst und sich zu verflüssigen beginnt. Im Innern sind bereits mehrere Zellkomplexe zu sehen, deren Kerne noch gut erhalten sind, deren Protoplasma aber fast aufgelöst ist und dem Inhalt das fädige Aussehen verleiht. Vergrößerung: Hartnack, L. 4, Ocular 3. Technik: Hämatein-van Gieson.

\title{
Literatur.
}

1. Hertwig, Entwicklungsgeschichte.

2. Verworn, Allgemeine Physiologie.

3. Georg Wegner, Über Lymphangiome. Langenbecks Archiv, Band XX. S. 641 .

4. Roth, Über Mißbildungen im Bereiche des Ductus omphalo-mesentericus. Dieses Archiv, Band 86, Heft III.

5. Runkel, Uber cystische Dottergangsgeschwülste. Inaug.-Diss. Marburg 1897.

6. Hackspill, Ein seltener Fall von Fibromyxoma cavernosum mesenterii. Inaug.-Diss. Würzburg 1898.

7. Penkert, Vier seltene Fälle von abdominalen Cysten. Deutsche Zeitschrift für Chirurgie. Band 64. Heft $\mathrm{V}$ und VI.

8. Nager, Beitrag zur Kenntnis seltener Abdominaltumoren. Beiträge zur allg. u. spez. Pathologie. Dr. E. Ziegler. Band 36. Heft I.

\section{XIX. \\ Ein Beitrag zur Genese der mesenterialen Chylangiome.}

\author{
Von \\ Dr. med. Paul Klemm, Riga, \\ dirigierendem Arzt der chirurgischen Abteilung des städtischen Kinder- \\ krankenhauses, Oberarzt des Roten Kreuzes.
}

Obgleich sich in den letzten Jahren die Mitteilungen von Fällen mesenterialer Chyluscysten gemehrt haben, so ist die Kasuistik dieser interessanten Tumorform doch noch immer so 